\title{
Differential expression of cyclin-dependent kinases in the adult human retina in relation to CDK inhibitor retinotoxicity
}

\author{
Phillip Wright $^{1} \cdot$ Janet Kelsall ${ }^{2} \cdot$ Guy Healing ${ }^{2,3} \cdot$ Julie Sanderson $^{1}$ (I)
}

Received: 10 October 2018 / Accepted: 10 December 2018 / Published online: 8 January 2019

(c) The Author(s) 2019

\begin{abstract}
Cyclin-dependent kinases (CDKs) are a family of kinases associated predominantly with cell cycle control, making CDK inhibitors interesting candidates for anti-cancer therapeutics. However, retinal toxicity (loss of photoreceptors) has been associated with CDK inhibitors, including the pan-CDK inhibitor AG-012896. The purpose of this research was to use a novel planar sectioning technique to determine CDK expression profiles in the ex vivo human retina with the aim of identifying isoforms responsible for CDK retinotoxicity. Four CDK isoforms (CDK11, 16, 17 and 18) were selected as a result of $\mathrm{IC}_{50}$ data comparing neurotoxic (AG-012986 and NVP-1) and non-neurotoxic (dinaciclib and NVP-2) CDK inhibitors, with $\mathrm{IC}_{50} \mathrm{~s}$ at CDK11 showing a clear difference between the neurotoxic and non-neurotoxic drugs. CDK11 was maximally expressed in the photoreceptor layer, whereas CDK16, 17 and 18 showed maximal expression in the inner nuclear layer. $C D K 5$ (an isoform associated with retinal homeostasis) was maximally expressed in the retinal ganglion cell layer. Apart from $C D K 18$, each isoform showed expression in the photoreceptor layer. The human Müller cell line MIO-M1 expressed CDK5, 11, 16 and 17 and AG-01298 $(0.02-60 \mu \mathrm{M})$ caused a dose-dependent increase in MIO-M1 cell death. In conclusion, CDK11 appears the most likely candidate for mediation of photoreceptor toxicity. RNA profiling can be used to determine the distribution of genes of interest in relation to retinal toxicity in the human retina.
\end{abstract}

Keywords CDK $\cdot$ Human $\cdot$ Retina $\cdot$ Müller cell $\cdot$ Retinotoxicity $\cdot$ CDK inhibitors

\section{Introduction}

Cyclin-dependent kinases (CDKs) are a large family $(>20)$ of serine/threonine protein kinases which play fundamental and complex roles within the cell (Malumbres 2014; Malumbres and Barbacid 2005). They are primarily associated with driving the cellular processes and signals which coordinate progression through the cell cycle. CDKs, as the name suggests, depend on cyclins for activation, forming heterodimeric complexes to enable activity. Cyclins are synthesised and degraded throughout the cell cycle which regulates the activity of CDKs with well-documented roles

Julie Sanderson

j.sanderson@uea.ac.uk

1 School of Pharmacy, University of East Anglia, Norwich NR4 7TJ, UK

2 AstraZeneca, Alderley Park, Macclesfield SK10 4TF, UK

3 Apconix Ltd, Biohub at Alderley Park, Cheshire, Macclesfield SK10 4TG, UK for CDKs 1, 2, 4 and 6 (Malumbres 2014; Malumbres and Barbacid 2005). Interestingly, CDK expression is present in post-mitotic cells and CDKs are known to play roles outside of the cell cycle (Hydbring et al. 2016; Lim and Kaldis 2013) including control over other important cell events such as differentiation and cell death. They also have a central involvement in transcriptional regulation, for example, CDKs 7, 8 and 9 regulate the activity of RNA polymerase II (Bregman et al. 2000; Malumbres 2014; Parry et al. 2010). CDK11 is involved in transcription and RNA processing events (Hu et al. 2003), but also is implicated in other cellular processes including autophagy (Wilkinson et al. 2011). In post-mitotic cells, much research has focussed on CDK5, which is atypical in that it is not activated by cyclins, is cytoplasmic/plasma membrane associated rather than nuclear and also has its highest expression in neurons, classical terminally differentiated cells. In neurons, CDK5 is associated with synaptic vesicle turnover, neurotransmitter release, post-synaptic signalling, synaptogenesis and synaptic plasticity, as well as regulation of cell survival/cell death pathways (Su and Tsai 2011). It is also implicated in the 
regulation of phototransduction in the retina (Hayashi et al. 2000) and is involved in retinal development (Hirooka et al. 1996; Nakayama et al. 1999). CDKs 16, 17 and 18 are within the CDK5 family (Malumbres 2014) and are also known to be expressed in neurons (Shimizu et al. 2014; Hirose et al. 1997; Herskovits and Davies 2006), although their role has been less well defined.

Recently, CDK inhibitors have become of interest due to their potential to be used as cancer therapeutics ( $\mathrm{Li}$ et al. 2016). Given their fundamental role in cell cycle control, CDK dysregulation is a common feature in cancer cells and they are over-expressed in many tumours (Nemunaitis et al. 2013). First-generation CDK inhibitors were pan-selective (Li et al. 2016) and these were effective in pre-clinical studies, with potent anti-proliferative activity. However, as might be expected given the extensive roles of the CDK family, they were associated with toxicity. For example, the pan-CDK inhibitor AG012986 was found to be highly neurotoxic, with both peripheral (sciatic nerve toxicity) and central (retinotoxicity) effects found in mice (Illanes et al. 2006). Clearly, such toxicity would limit therapeutic use; whether such toxicity would be expected to occur in humans is a key consideration. It is also important to understand which CDKs are involved in mediating neurotoxicity to predict whether more selective inhibitors would exhibit similar neurotoxic effects. Second-generation CDK inhibitors have been developed which have greater selectivity and some are showing therapeutic potential, with numerous clinical trials ongoing. One drug, palbociclib (a CDK4/6 inhibitor), has been FDA approved, albeit with restricted clinical indications (Zhang et al. 2017). Highly selective CDK9 inhibitors are also of interest as cancer therapeutics (Franco et al. 2018), with proposed mechanisms via the regulation of RNA Polymerase II (Huang et al. 2014; Lu et al. 2015; Okuda et al. 2016; Rahl et al. 2010; Samarakkody et al. 2015). NVP-1 and NVP-2 are selective CDK9 inhibitors (Barsanti 2011) which, interestingly, display differential neurotoxicity (Sutton 2014). Other inhibitors, for example dinaciclib, have a wider selectivity, which may prove important in terms of efficacy given the significant redundancy observed in CDKs in relation to cell cycle control (Di Giovanni et al. 2016).

The retina is responsible for phototransduction, transforming light energy into neuronal signals which are in turn processed by the brain to enable vision. The retina is divided into layers including three distinct nuclear layers (the inner and outer nuclear layer and the ganglion cell layer) and two synaptic layers (the inner and outer plexiform layers) (Fig. 1). The ganglion cell layer is dominated by retinal ganglion cells which are responsible for the transmission of information from the retina to the brain (Sanes and Masland 2015). The axons of the retinal ganglion cells make up the nerve fibre layer (the innermost retinal layer) and leave the eye at the optic nerve head. The next nuclear layer is the

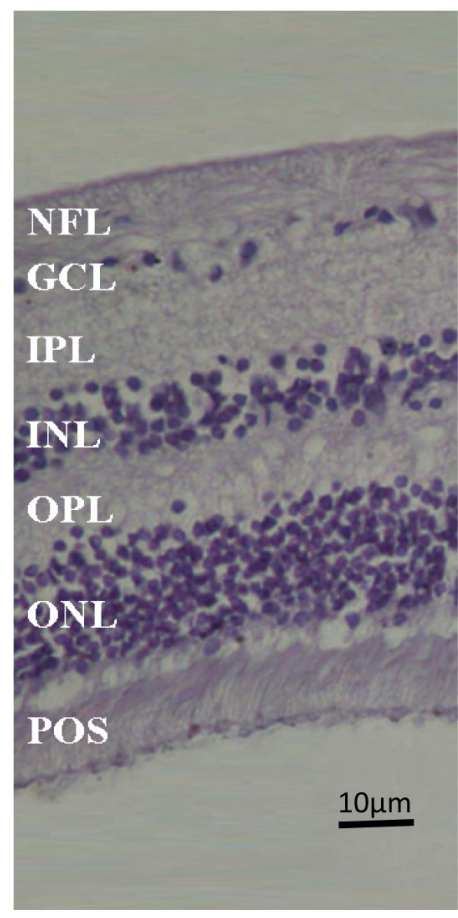

Fig. 1 Light micrograph of human retina. NFL nerve fibre layer, $G C L$ ganglion cell layer, $I P L$ inner plexiform layer, $I N L$ inner nuclear layer, $O P L$ outer plexiform layer, $O N L$ outer nuclear layer, $P O S$ photoreceptor outer segments

inner nuclear layer which consists of the cell bodies of several retinal cells including Müller cells (glial cells responsible for physical and metabolic support of the retinal neurons) (Bringmann et al. 2006; Reichenbach and Bringmann 2013), bipolar cells (transmit signals from the photoreceptors to the ganglion cells) (Euler et al. 2014), horizontal cells (modulate signalling between photoreceptors and bipolar cells) (Poche and Reese 2009), amacrine cells (provide inhibitory modulation of information to bipolar cells) (Forrester 2007), interplexiform neurons (provide long range feedback with processes which extend into both the inner plexiform layer and the outer plexiform layer) (Jiang and Shen 2010) and some displaced ganglion cells. The most posterior nuclear layer is the outer nuclear layer which consists of cell bodies from rod and cone photoreceptors, the cells responsible for converting light into a neural signal (Forrester 2007).

The aim of these experiments was to investigate CDK expression in the human retina. Based on $\mathrm{IC}_{50}$ data, predictions were made regarding the CDKs which may be involved in neurotoxicity. The expression of these was investigated in the human retina using a novel mRNA expression profiling technique, and the results compared known retinal cell markers to determine regions of high expression. It also aimed to investigate toxicity of AG-012986 in human Müller cells in relation to CDK expression. Revealing the localisation of CDK expression within the retina may help determine 
the isoform/s responsible for the retinal toxicity found with the CDK inhibitor AG-012986 and help inform the future development of CDK inhibitors to avoid retinotoxicity. The development and characterisation of such techniques may also prove useful in future investigations of other targets relating to retinal toxicity.

\section{Materials and methods}

\section{CDK inhibitor affinity}

The CDK inhibitors NVP-1, NVP-2, dinaciclib and AG012986 were synthesised in house by AstraZeneca. The $\mathrm{IC}_{50} \mathrm{~s}$ of the CDK inhibitors towards the CDKs of interest were assessed by DiscoverX (Eurofins DiscoverX Corporation, Fremont, USA) by means of their KINOMEscan profiling service. KINOMEscan utilizes three components which are combined to form a competition binding assay: DNAtagged kinase, immobilized ligand and the CDK inhibitor of choice. The ability of the CDK inhibitor to compete with the immobilized ligand is then assessed via qPCR, as described by Fabian et al. (2015).

\section{Human retinal explant dissection}

The East Anglian Eye Bank provided donor human eyes within $24 \mathrm{~h}$ post mortem. The cornea had been removed for transplantation and the remainder had consent for research. The research was conducted with full ethical approval under the tenets of the declaration of Helsinki. Only eyes with no known retinal pathology/injury were utilised for research. In total, 16 post mortem donor eyes were used in this study from donors aged between 41 and 92 years old. The retina was dissected as described previously (Niyadurupola et al. 2011; Osborne et al. 2016). Briefly, the lens and the iris were removed by a circumferential incision. The weight of the vitreous was then used to detach the retina from the RPE and an incision made around the optic nerve head to fully detach the retina and vitreous from the RPE and sclera. The vitreous was detached from the retina and small incisions made at periphery to allow the retina to flatten. The retinal preparation was placed over an extraction template (Osborne et al. 2016) using the optic nerve and fovea as reference points, and the macula and five paramacular explants removed using a $4 \mathrm{~mm}$ trephine (Biomedical Research Instruments, MD, USA). Paramacula sample 3 (temporal to the macula) was used for explant analysis. The explants were either snap frozen in liquid nitrogen for RNA extraction or prepared for planar sectioning.

\section{Planar retinal sectioning}

Planar sectioning was performed as described previously (Niyadurupola et al. 2013). Briefly, macular and paramacular explants were dissected from donor eyes, removed from the culture dish using filter paper and mounted onto a flattened surface of frozen optimal cutting temperature compound (OCT) (Sakura Finetek, Zoeterwoude, Netherlands). Another layer of OCT was then applied on top of the mounted retinal explant and frozen. A Bright OTF 5000 cryostat (Bright instruments, Huntingdon, UK) was used to cut $20 \mu \mathrm{m}$ sections which were collected in $1.5 \mathrm{ml}$ Eppendorfs, snap frozen in liquid nitrogen and stored at $-80^{\circ} \mathrm{C}$.

\section{Quantitative real-time PCR (qRT-PCR)}

RNA was extracted from retinal explants using the RNeasy Mini Kit (Qiagen, Crawley, UK) or planar sections using the RNeasy Micro Kit (Qiagen, Crawley, UK). The concentration and quality of RNA was assessed using a Nanodrop ND-1000 spectrophotometer (Nanodrop Technologies, Wilmington, USA). The RNA was then reverse transcribed to cDNA using Superscript ${ }^{\mathrm{TM}}$ II, dNTP mix and random primers (Invitrogen, Paisley, UK). 5 ng of cDNA was mixed with Mastermix (Applied Biosystems, Warrington, UK) plus probes/primers (see Table 1) and mRNA expression assessed using Taqman qRT-PCR (ABI Prism 7700 Sequence Detection System; Applied Biosystems, Warrington, UK). Expression in explants was normalised to the geometric mean of the house-keeping genes topoisomerase DNA I (TOPI) and cytochrome c-1 (CYC1) (Niyadurupola et al. 2011). Since the expression of house-keeping genes varied across the retina, expression in planar sections was normalised to the section with the highest expression (Niyadurupola et al. 2013). Expression in samples from different donors was aligned by superimposing the expression profile for $R C V R N$.

\section{Cell culture}

The human Müller cell line (MIO-M1) was kindly provided by Professor Astrid Limb (University College London, UK) and is derived from a primary culture of human retinal cells (Limb et al. 2002). MIO-M1 cells were routinely cultured in DMEM GLUTAMAX medium (ThermoFisher, Loughborough, UK) with 10\% FBS (ThermoFisher, Loughborough, UK), and $50 \mu \mathrm{g} / \mathrm{l}$ Pen-Strep (ThermoFisher, Loughborough, UK) in a humidified incubator $\left(35{ }^{\circ} \mathrm{C} ; 95 \%\right.$ air; $5 \%$ $\mathrm{CO}_{2}$ ). For assessment of cell viability/cell death, cells were cultured in 96 well plates (ThermoFisher, Loughborough UK). Cells were plated in serum-supplemented medium to reach $90 \%$ confluency, then serum-starved for $24 \mathrm{~h}$ prior to exposure to experimental conditions. AG012986 was dissolved in DMSO and diluted in SF medium to the desired 
Table 1 List of PCR primers/ probes

\begin{tabular}{llll}
\hline Primer/probe & Number & Reporter & Source \\
\hline Topoisomerase (TOP1) & & FAM & Primer Design, Southampton, UK \\
Cytochrome C1 (CYC1) & & FAM & Primer Design, Southampton, UK \\
THY1 & Hs00174816_m1 & FAM & Applied Biosystems, Warrington, UK \\
Protein kinase C alpha & Hs00925193_m1 & FAM & Applied Biosystems, Warrington, UK \\
Choline acetyltransferase & Hs00252848_m1 & FAM & Applied Biosystems, Warrington, UK \\
Calbindin & Hs01077197_m1 & FAM & Applied Biosystems, Warrington, UK \\
Retinaldehyde binding protein 1 & Hs00165632_m1 & FAM & Applied Biosystems, Warrington, UK \\
Recoverin & Hs00610056_m1 & FAM & Applied Biosystems, Warrington, UK \\
Cyclin-dependent kinase 5 & Hs00358991_g1 & FAM & Applied Biosystems, Warrington, UK \\
Cyclin-dependent kinase 11A/B & Hs02341397_m1 & FAM & Applied Biosystems, Warrington, UK \\
Cyclin-dependent kinase 16 & Hs00178837_m1 & FAM & Applied Biosystems, Warrington, UK \\
Cyclin-dependent kinase 17 & Hs00176839_m1 & FAM & Applied Biosystems, Warrington, UK \\
Cyclin-dependent kinase 18 & Hs00384387_m1 & FAM & Applied Biosystems, Warrington, UK \\
\hline
\end{tabular}

concentration with a DMSO content of less than $0.1 \%$ and incubated with the cells for $24 \mathrm{~h}$.

\section{Assessment of cell death and cell viability}

Following incubation in experimental conditions for $24 \mathrm{~h}$, $100 \mu \mathrm{l}$ of media was removed for analysis of cell death using the LDH assay according to manufacturer's instructions (Roche, Burgess Hill, UK). The absorbance was measured at $490 \mathrm{~nm}$ (BMG labtech platereader, Aylesbury, UK). Background was subtracted and data expressed as fold-change relative to control levels. To assess cell viability, the remaining medium was aspirated and cell viability was assessed using the CellTiter $96^{\circledR}$ AQueous One Solution Cell Proliferation Assay (MTS assay) (Promega, Southampton, UK). The assay was performed according to the manufacturer's instructions and the plate measured at $490 \mathrm{~nm}$ (BMG labtech platereader, Aylesbury, UK). Background was subtracted and data expressed relative to control (\%). Four independent replicates of each experiment were carried out.

\section{Results}

\section{Selectivity of four CDK inhibitors to CDK isoforms}

Initial experiments compared the $\mathrm{IC}_{50} \mathrm{~s}$ of the CDK inhibitors NVP1, NVP2, dinaciclib and AG-012986 for a range of CDKs (Fig. 2). These inhibitors were selected due to their neurotoxicity profiles; both AG-012986 and NVP-1 have reported neurotoxicity (in vivo; mouse) (Illanes et al. 2006; Sutton 2014), whereas NVP-2 and dinaciclib have no reported neurotoxicity. The neurotoxic inhibitor NVP-1 displayed an approximate tenfold lower $\mathrm{IC}_{50}$ for CDKs 11, 16, 17 and 18 compared to the non-neurotoxic NVP-2. This implicates these CDK isoforms in mediation
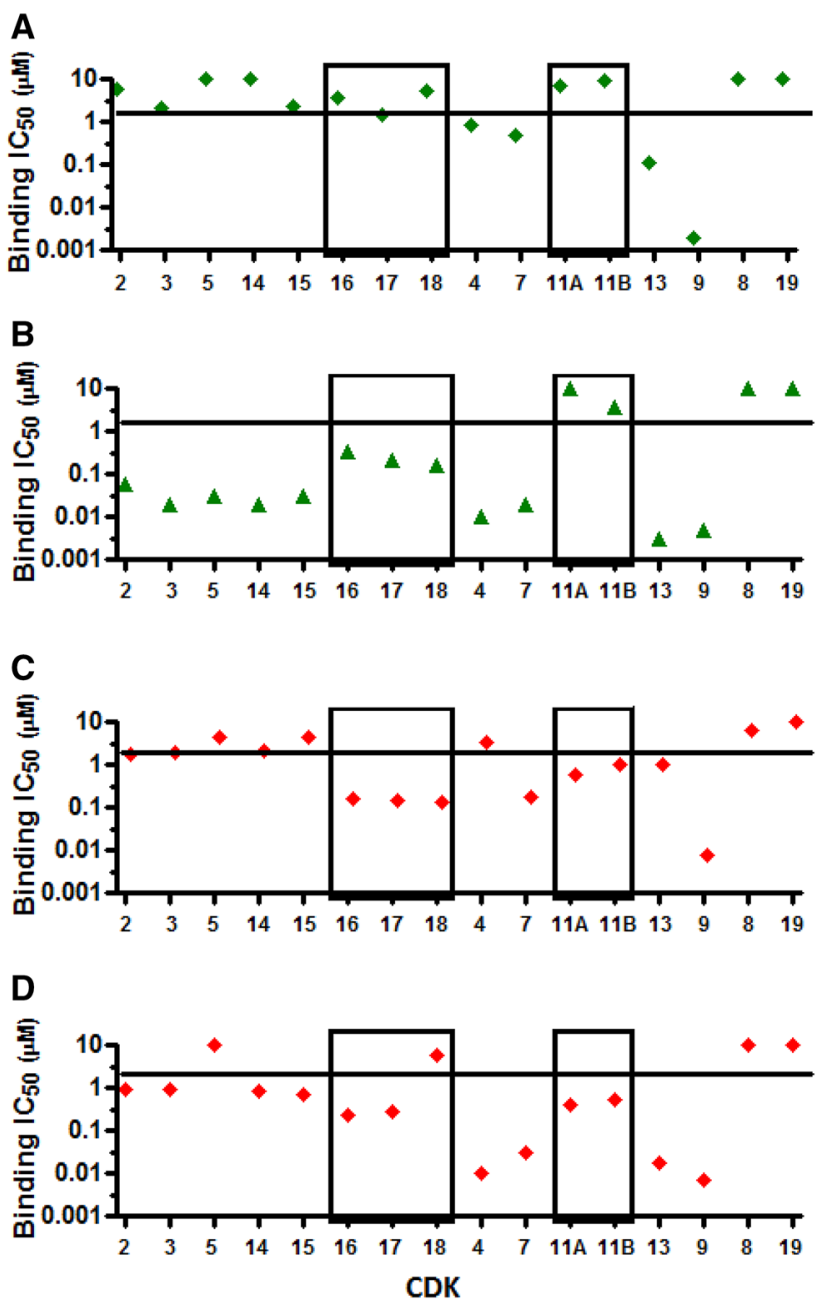

Fig. $2 \mathrm{IC}_{50} \mathrm{~s}$ of four CDK inhibitors towards different CDK isoforms. Green symbols (A and $\mathbf{B}$ ) represent two CDK inhibitors without any recorded neurotoxic side effects, red symbols $(\mathbf{C}$ and $\mathbf{D})$ represent two CDK inhibitors with recorded neurotoxicity. A NVP-2, B dinaciclib, C NVP-1, D AG-012986. (Color figure online) 
of neurotoxicity. Interestingly, AG-012986, which is also neurotoxic, possesses a similar $\mathrm{IC}_{50}$ to NVP-2 for CDK 11, 16 and 17. Dinaciclib, which has no reported neurotoxicity, had a markedly different profile to the other CDK inhibitors investigated, with high potency $\left(\mathrm{IC}_{50}<100 \mathrm{nM}\right)$ for all of the CDKs tested apart from CDKs 11, 16, 17 and 18 (identified above) plus CDKs 8 and 19.

These data indicate that CDK11 may be the most interesting isoform to investigate as a possible candidate for mediation of retinotoxicity, with CDK 16, 17 and 18 also of potential interest; these CDKs were therefore selected for further investigation. CDK5 was also investigated further due to its known roles in supporting neuronal and RGC survival (Cheung et al. 2008), its involvement in RGC death in glaucoma (Chen et al. 2011) and potential role in phototransduction in the retina (Matsuura et al. 2000).

\section{Expression of retinal cell markers in macula and paramacula explants}

Retinal ganglion cells (RGCs) are found at highest abundance in the macular region of the retina. Accordingly, the RGC marker THY1 (Fig. 3A) displayed significantly higher expression in macula samples compared to the paramacula.
Conversely, rod ON bipolar cells are in low abundance in the macula and the marker for these cells, PRKCA (Fig. 3B) displayed significantly lower expression in the macula compared to the paramacula. The horizontal cell marker $C A L B 1$ and the amacrine cell marker CHAT (Fig. 3C, D, respectively) displayed little difference in expression between the paramacula and macula samples, whereas the Müller cell marker RLBPI (Fig. 3E) showed significantly lower expression in the macula. The marker of photoreceptors RCVRN (Fig. 3F) showed no significant difference in expression between the paramacula and macula, however, a trend of lower expression in the macula was found. The distributions of retinal cell markers were consistent with the known distribution of retinal cells in the macular and paramacular retina, demonstrating that this technique could be useful to indicate the distribution of the CDKs of interest within retinal cells.

CDK5, 11, 16, 17 and 18 transcripts were detected in the human retina (Fig. 4). When comparing expression between the macula and paramacula, $C D K 5$ and $C D K 18$ displayed differences in expression between the two regions, with CDK5 showing greater expression in the macula (Fig. 4A) and $C D K 18$ showing the opposite distribution (Fig. 4E). These data suggest that CDK5 and 18 are differentially distributed in the retina which may relate to expression being
A: THY1

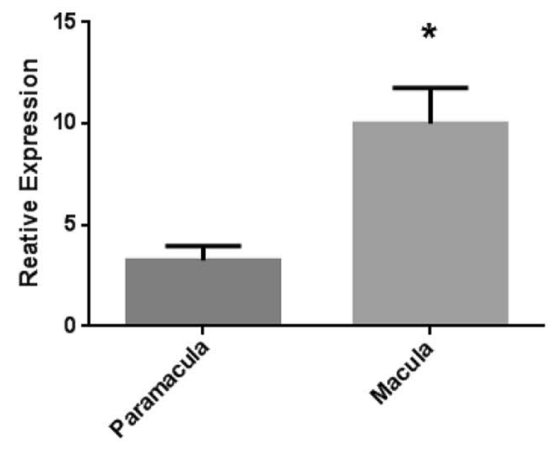

D: $C H A T$

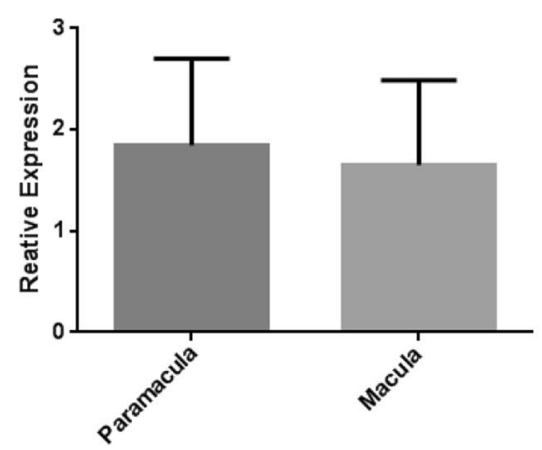

B: PRKCA

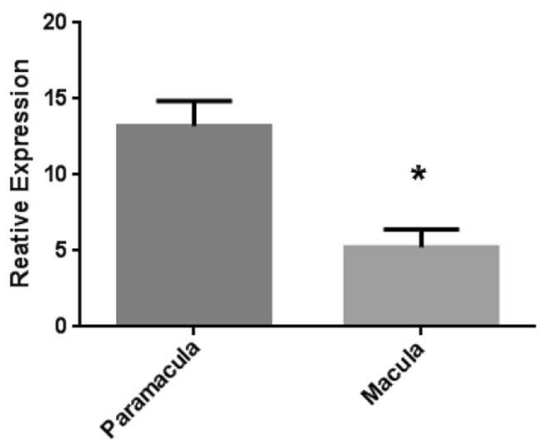

E: RLBP1

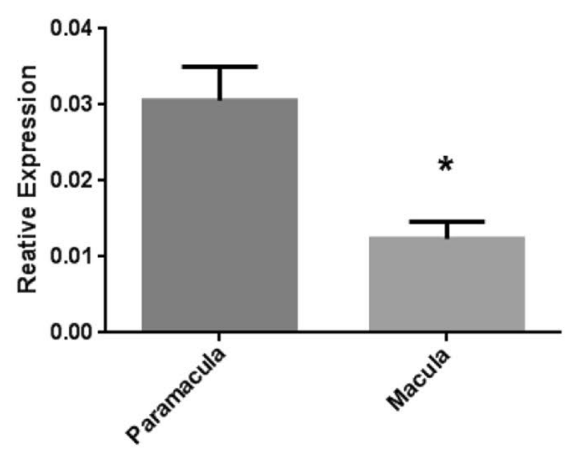

C: CALB1

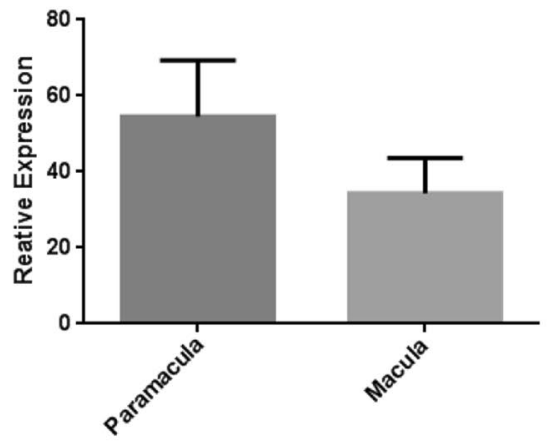

$\mathrm{F}: \operatorname{RCVRN}$

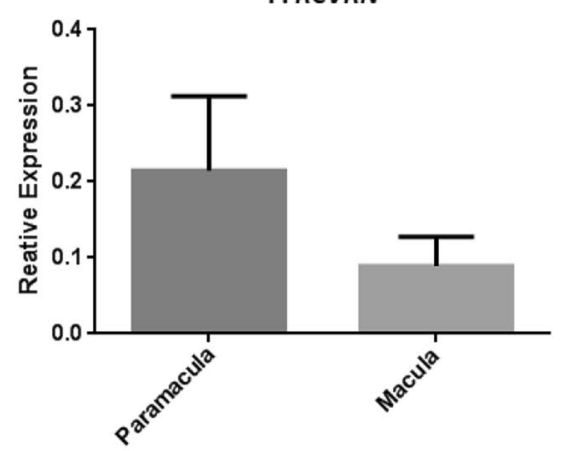

Fig. 3 Expression of A THY1, B PRKCA, C CALB1, D CHAT, E RLBP1, and F RCVRN mRNA in human macula and paramacula retina relative $T O P 1$ and $C Y C 1$. Mean $\pm \operatorname{SEM}(n=4) *$ significant difference from the control (unpaired $T$ test $)(P<0.05)$ 
A: CDK5

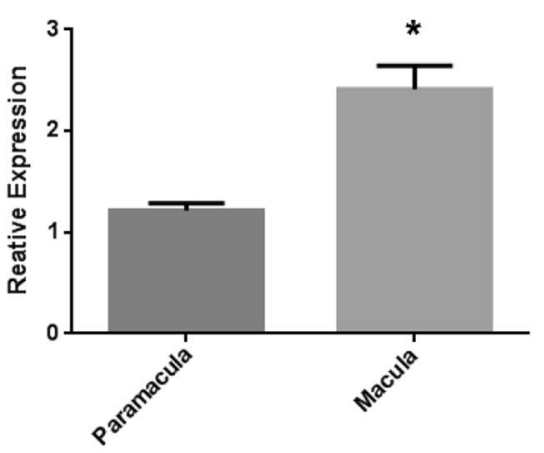

E: $C D K 17$

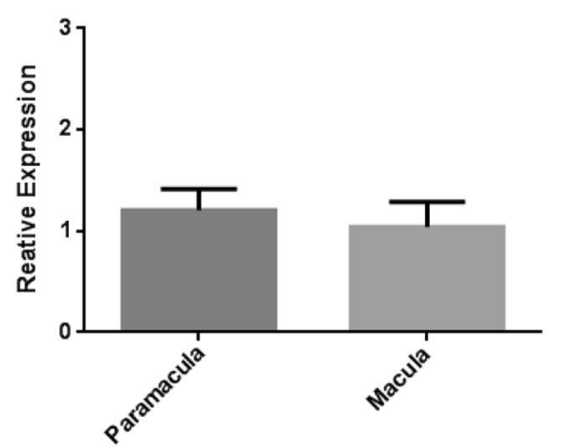

B: $C D K 11$

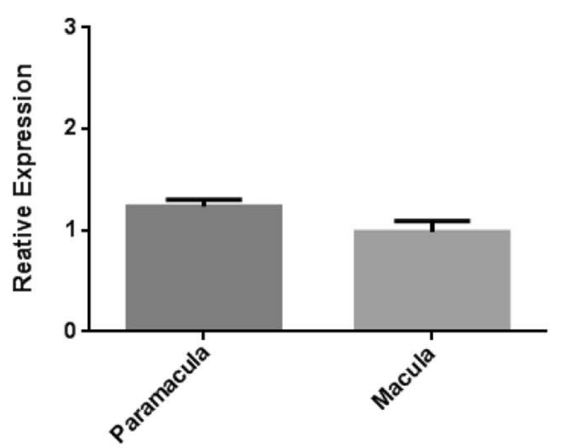

F: $C D K 18$

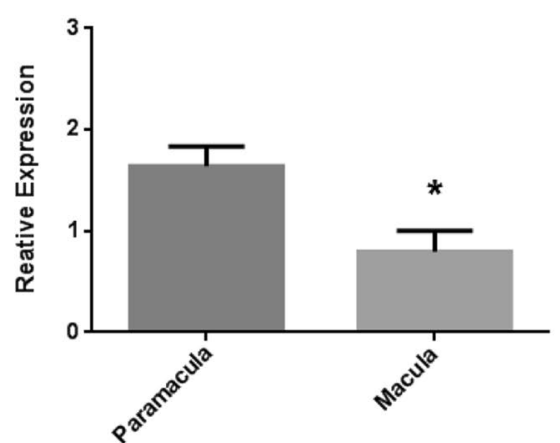

C: $C D K 16$

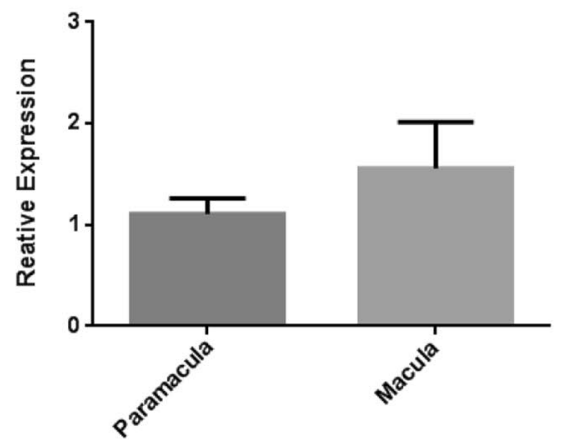

Fig. 4 Expression of A $C D K 5$, B $C D K 11, \mathbf{C} C D K 16, \mathbf{D} C D K 17$ and $\mathbf{E} C D K 18$ mRNA in human macula and paramacula retina relative to housekeeping genes TOP1 and $C Y C 1$. Mean $\pm \operatorname{SEM}(n=4) *$ significant difference from the control (unpaired $T$ test $)(P<0.05)$

localised to different cell types that show similar distributions (Fig. 3).

\section{Expression profiles of CDKs compared to cell-specific markers in the macula and paramacula human retina}

To investigate the distribution of CDK transcripts within the layers of the human retina, expression profiling was used (Niyadurupola et al. 2013). The distribution of cell specific markers was first determined in planar-sectioned macula and paramacula retina (Fig. 5). Peak expression of THYI was observed in the inner retina in both macula and paramacula retina, corresponding to expression in the ganglion cell layer, as would be anticipated for a RGC marker. In the mid-section, corresponding to the inner nuclear layer (INL), there was peak expression of the markers $R L B P 1, C A L B 1$ and $P R K C A$. Expression of these markers was similar in both macula and paramacula samples and displayed low levels of expression in the outer retina, reaching peak expression in the inner nuclear layer, followed by a decrease in expression through the inner retina (GCL). CHAT expression was baseline in the outer retina of both macula and paramacula samples. However in the macula, peak expression was seen in sections associated with INL, whereas peak expression in the paramacula explant did not occur until the ganglion cell layer. As would be anticipated for a marker of photoreceptors, the outermost retina, corresponding to the outer nuclear layer (ONL), displayed highest expression of $R C V R N$ both in the macula and paramacula sections. Peak expression in the ONL was followed by a decrease towards the INL and baseline expression in the GCL.

The expression profile of the CDKs of interest was determined in relation to the distribution of the known retinal cell markers. Figure 6 shows the macula and paramacula expression profiles of CDK5, 11, 16, 17 and 18 . Profiles of THY1, PRKCA and RCVRN in the same samples identify the ganglion cell layer, the inner nuclear layer and the outer nuclear layer, respectively. CDK5 expression peaked in the GCL of both the macula and paramacula retina. However, in the macula, there was little variation across the different layers, whereas, in the paramacula, expression was greater than twofold higher in the GCL compared to the outer layers. CDK11 displayed peak expression in the ONL in both macula and paramacula retina, with expression being approximately twofold and fourfold higher in the ONL compared to the GCL in the macula and paramacula, respectively. CDK16 displayed low expression in the outer nuclear layer of the macula, followed by increasing expression in the inner nuclear 
Fig. 5 Expression profiles of THY1, CHAT, RLBP1, CALB1, $P R K C A$ and $R C V R N$ in macula and paramacula human retina. The outer nuclear layer is represented by the light grey bar, the inner nuclear layer by the medium grey bar and the ganglion cell layer by the dark grey bar. Mean $\pm \operatorname{SEM}(n=4)$

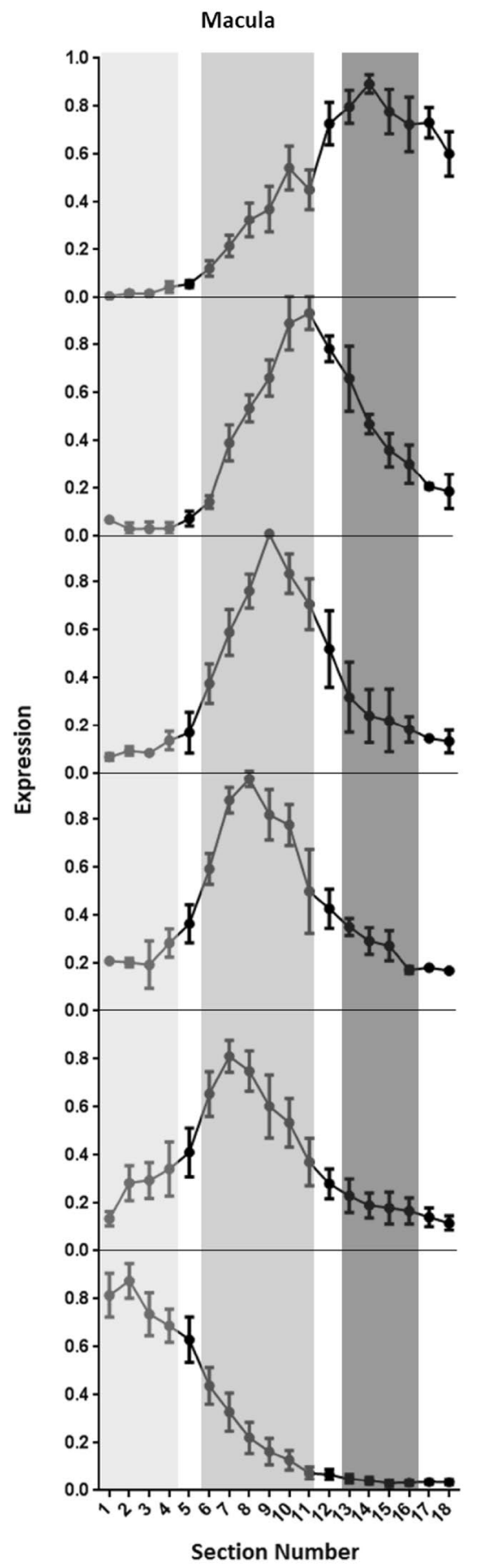

Paramacula

THY1

CHAT

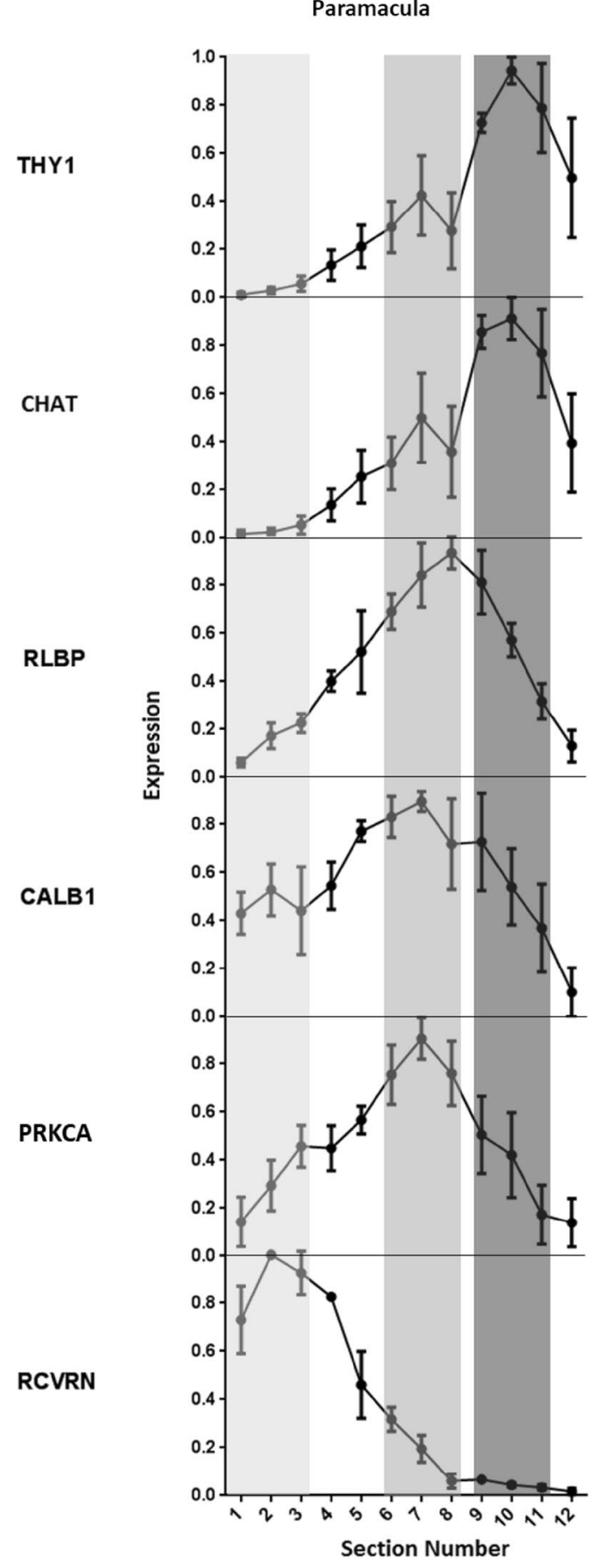

layer and remaining high throughout the GCL. In the paramacula, there was also low expression in the outer nuclear layer, however, this was followed by peak expression in the inner nuclear layer and low expression in the ganglion cell layer. $C D K 17$ and $C D K 18$ displayed a similar pattern of expression in both macula and paramacula samples, with low expression in the ONL (close to no expression with $C D K 18$ ), followed by peak expression in the INL, and low expression in the GCL.

\section{Effect of the CDK inhibitor AG-012986 on Müller cell viability}

To investigate retinal cell toxicity, the effects of the CDK inhibitor AG-012986 on Müller cell viability was investigated using the MIO-M1 cell line (Limb et al. 2002). In addition, expression of CDK5, 11, 16, 17 and 18 was assessed. It was found that AG-012986 was toxic to MIOM1 cells, with a dose-dependent decrease in cell viability 
Fig. 6 Expression profile of CDK5, CDK11, CDK16, $C D K 17$ and $C D K 18$ mRNA in macula and paramacula samples. Profiles of THY1, PRKCA and $R C V R N$ are shown for comparison. The outer nuclear layer is represented by the light grey bar, the inner nuclear layer by the medium grey bar and the ganglion cell layer by the dark grey bar. Mean $\pm \operatorname{SEM}(n=4)$

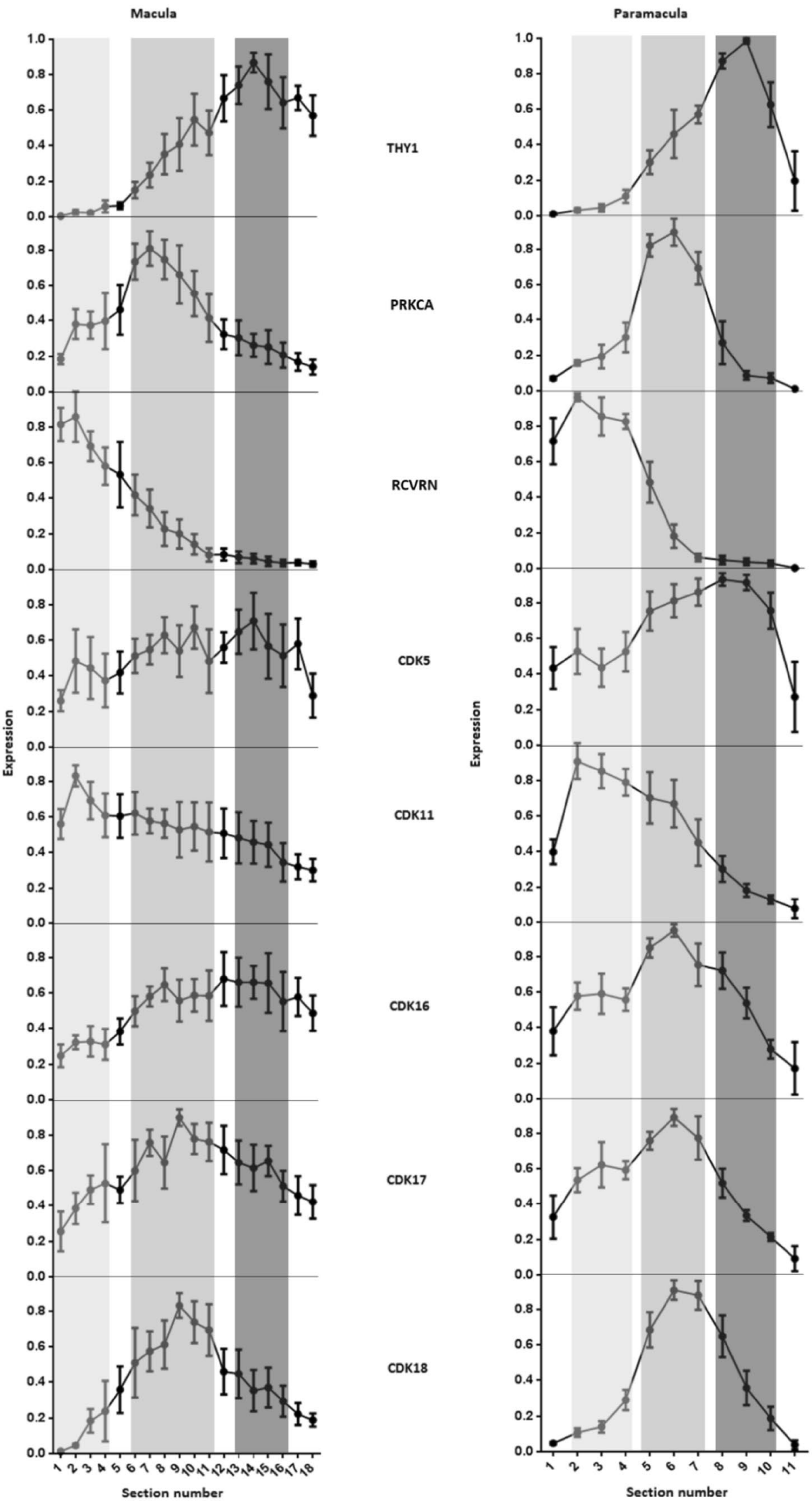


detected using the MTS proliferation assay, and a corresponding increase in LDH release (Fig. 7). In each case, a significant effect was achieved at a concentration of $200 \mathrm{nM}$ AG-012986, and a maximal response achieved at $500 \mathrm{nM}$. The maximal decrease in viability was approximately $25 \%$, i.e., full toxicity was not observed. MIO-M1 cells expressed $C D K 5,11,16$ and 17 , but there was very little expression of CDK18 (Fig. 8).

\section{Discussion}

Cyclin-dependant kinases are potential targets for cancer therapeutics and exploitation of CDK inhibitors is of major interest. Chemotherapy drugs that target rapidly dividing cancer cells have detrimental side effects on rapidly dividing non-cancer cells. This is true for CDK inhibitors (Lee and Jessen 2012), for example, leukopenia is a dose-limiting factor for dinaciclib (Mita et al. 2014; Stephenson et al. 2014) and flavopiridol (Luke et al. 2012; Jones et al. 2014). However, the pan-CDK inhibitor AG-0129896 also showed an unexpected toxic effect, with sciatic nerve and photoreceptor toxicity being found when AG-012986 toxicity was assessed in mice (Illanes et al. 2006). Interestingly, both of these cell types have an arrested cell cycle, yet are susceptible to the effects of CDK inhibition. Potential toxicity is an important consideration when developing CDK inhibitors as cancer therapies; a better understanding of mechanisms would be beneficial.

Initial experiments determined which CDKs were the most interesting in terms of mediation of neurotoxicity. Four different CDK inhibitors were compared, two had reported neurotoxicity, whereas two had no reported neurotoxicity. Their $\mathrm{IC}_{50} \mathrm{~s}$ for a range of CDKs were assessed. Two CDK families of interest were identified: the CDC2L family

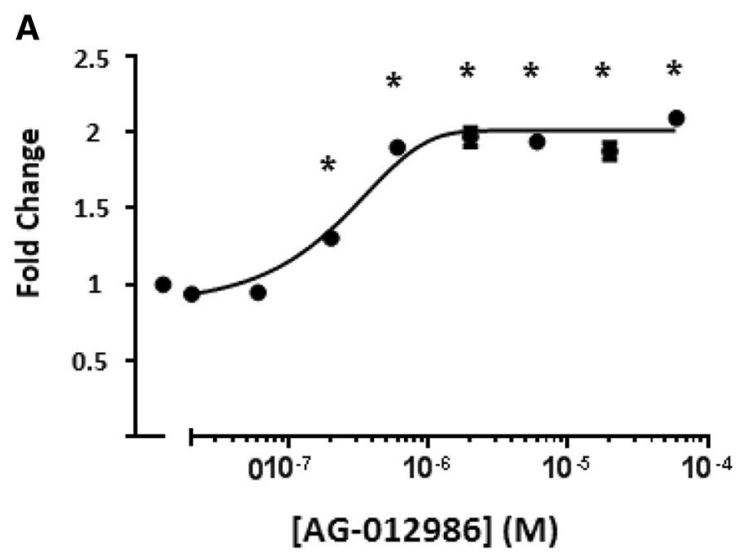

Fig. 7 LDH release and cell viability of MIO-M1 cells in response to 24 h treatment with AG-012986. a LDH release from MIO-M1 cells in response to the pan-CDK inhibitor AG-012986. b \% control cell

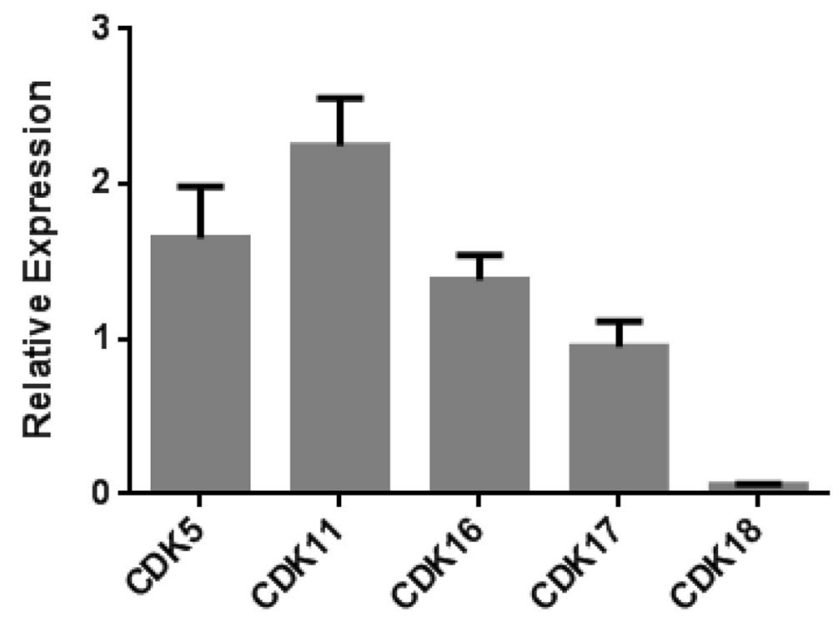

Fig. 8 Expression of $C D K 5, C D K 11, C D K 16, C D K 17$ and $C D K 18$ mRNA in MIO-M1 cells relative to TOP1 and $C Y C 1$. Mean \pm SEM $(n=3)$

(CDK11A and 11B) and the PCTK family (CDK16, 17 and 18). CDK5 was also investigated due to its well-documented role in neuronal homeostasis (Su and Tsai 2011).

To investigate localization of CDKs in the human retina, the expression of known retinal cell markers was first characterised. Expression of cell-specific markers was investigated topographically in macula and paramacula retina, followed by determination of the expression profiles of the cellular markers in planar-sectioned macula and paramacula retina. Whole explant mRNA analysis of the cell-specific markers showed the ganglion cell marker THYl to have significantly higher expression in the macula compared to the paramacula. RGCs are present at a higher density in the macula compared to paramacula explants (Niyadurupola et al. 2011), demonstrating that this technique can provide information regarding the distribution of retinal cell types.

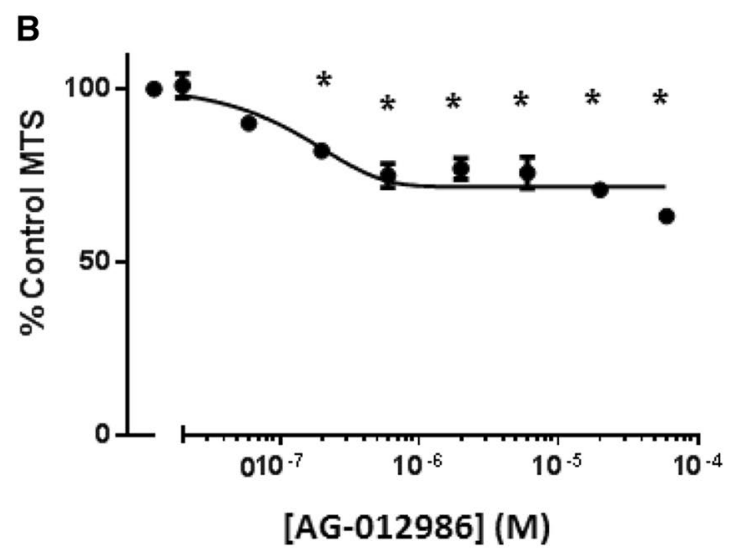

viability of MIO-M1 cells in response to differing concentrations of AG-012986. *Significant difference from the control (one way ANOVA with Dunnet's post hoc test $)(P<0.05)$ 
Planar sectioning confirmed peak expression of $T H Y-1$ to be localised to the GCL. The inner nuclear layer markers CHAT (amacrine cells) and CALB1 (horizontal cells) had equivalent expression in paramacula and macula retina, whereas the inner nuclear layer markers PRKCA (rod ON bipolar cells) and $R L B P$ (Müller cells) had significantly lower expression in the macula compared to the paramacula retina. The lower expression of $P R K C A$ in macula samples compared to paramacula samples can be explained by the location of rod ON bipolar cells. Rod ON bipolar cells only appear approximately $1 \mathrm{~mm}$ away from the fovea (Lameirao et al. 2009), resulting in fewer cells in the macula sample expressing PRKCA. The differential expression of the Müller cell marker $R L B P$ does not correspond directly to cell density, but may reflect differences in volume of the cells across the retina (Reichenbach and Bringmann 2013). Peak expression of all inner nuclear layer markers was localised to the inner nuclear layer apart from CHAT in the paramacula retina, where peak expression occurred more towards the inner retina. This is likely to reflect the occurrence of displaced CHAT-positive amacrine cells in the RGC layer. The marker of photoreceptors $(R C V R N)$ did not show a significant difference in expression between the macula and paramacula retina, although there was a trend towards lower expression in the macula. This corresponds to the differences in photoreceptor layer thickness seen in macula and paramacula human retinal explants (Niyadurupola et al. 2011). Planar sectioning revealed peak expression to occur in the outer nuclear layer corresponding with the known distribution of photoreceptors. The distribution of cell-specific markers in the paramacula and macula retina indicated that these techniques are useful to assess distribution of genes of interest in the retina. Expression of the selected CDKs was therefore investigated in macula and paramacula retina and the expression profiles of CDKs of interest evaluated.

CDK5 showed significantly higher expression in the macula compared to the paramacula retina. This distribution indicates that $C D K 5$ may be expressed in ganglion cells, since THYI is the only marker that shows this distribution. mRNA profiling in paramacula samples indeed showed peak expression of $C D K 5$ to occur in the ganglion cell layer, although in the macula, expression was similar throughout all retinal layers. This suggests that CDK5 is expressed in human RGCs, but that expression also occurs in other cell types throughout the retina. This distribution is consistent with CDK5 protein expression in the retina, where the highest levels were found in the inner retina (Hayashi et al. 2000). The functions of CDK5 in the human retina remain to be fully elucidated, although there is evidence of a role in phototransduction (Hayashi et al. 2000) which could link CDK5 inhibition and retinotoxicity. However, dinaciclib had the highest binding affinity for CDK5 of the inhibitors tested and is not associated with neurotoxicity, indicating that
CDK5 is not a likely candidate for CDK inhibitor-mediated retinotoxicity. Interestingly, research has implicated CDK5 in RGC death in a rat model of glaucoma, where CDK5 upregulation was correlated with a significant increase in TUNEL positive cells. Roscovine (a CDK5 inhibitor) significantly reduced the number of apoptotic RGCs, emphasising a potential role for CDK5 in glaucomatous RGC cell death (Chen et al. 2011).

CDK11 expression was similar in macula and paramacula retina and planar sectioning revealed peak expression of CDK11 in the photoreceptor layer of both macula and paramacula samples, although relative differences were more noticeable in the paramacula retina. This indicates that $C D K 11$ is more highly expressed in photoreceptors and that expression of $C D K 11$ could be more associated with rod photoreceptors which are the predominant photoreceptors outside of the macula. This distribution, taken together with the binding affinity data, implicates CDK11 as the most likely candidate for mediation of AG-012986 photoreceptor toxicity. There are two highly homologous genes for CDK11 (CDK11A and CDK11B) and from each gene there is a full-length protein $\left(\mathrm{CDK} 11^{\mathrm{p} 110}\right)$ as well as several shorter isoforms including CDK11 ${ }^{\mathrm{p} 58}$ (Malumbres 2014). CDK11 biology is complex, with differing roles for different CDK11 isoforms. For example, CDK11 ${ }^{\mathrm{p} 110}$ is expressed continuously throughout the cell cycle, suggesting roles outside of mitotic control, and has been shown to be involved in transcriptional regulation and RNA processing (Hu et al. 2003), whereas the CDK11 $1^{\mathrm{p} 58}$ variant is specifically expressed during the $\mathrm{G} 2 / \mathrm{M}$ phase of the cell cycle and has roles including centriole duplication and spindle dynamics (Petretti et al. 2006), as well as being associated with cell cycle arrest and apoptosis (Rakkaa et al. 2014). CDK11 has also been associated with modulation of autophagy, with knockdown of CDK11 both inducing autophagy and impeding passage through the autophagic process (Wilkinson et al. 2011). CDK11 has not previously been studied within the retina. However, its expression in neuronal cells has previously been confirmed and a change in CDK11 cellular distribution has been associated with Alzheimer's disease (Bajic et al. 2011). In addition, CDK11 $1^{\mathrm{p} 58}$ was found to modulate apoptosis in PC12 cells (a rat neuronal cell line) with this isoform promoting apoptosis (Liu et al. 2013).

CDK16 expression showed no distinct pattern of expression between paramacula and macula explants. mRNA profiling of macula sections revealed increasing expression within the inner nuclear layer before reaching peak expression towards the inner retina, whereas in paramacula sections there was peak expression within the inner nuclear layer. $C D K 16$, is therefore expressed in the human retina, but cellular localization is unclear. CDK16, also known as Pctaire 1, is a member of the CDK5 family and has been shown to be expressed in brain, testis and skeletal muscle 
(Besset et al. 1999; Shimizu et al. 2014) and is implicated in control of exocytosis (Liu et al. 2006). Knockdown of CDK16 has previously been associated with induction of apoptosis in melanoma cells (Yanagi et al. 2014); its involvement in retinotoxicity should not be ruled out.

CDK17 expression was similar in the macula and paramacula retina. mRNA profiling of macula and paramacula samples showed low expression in the outer nuclear layer, with peak expression in the inner nuclear layer and decreasing expression in the ganglion cell layer, overall displaying a similar profile to CHAT and CALB1. These data indicate that amacrine and/or horizontal cells possess high levels of CDK17. CDK17, also known as Pctaire2, is a poorly characterised protein of the CDK5 family. It is expressed in terminally differentiated neurones with a suggested association with cytoskeletal proteins of post-mitotic neurones (Hirose et al. 1997), but its role remains undetermined.

CDK18 expression was lower in the macula compared to the paramacula retina and similar to the distribution of $P R K C A$ (bipolar cells) and RLBP (Müller cells). mRNA profiling of macula and paramacula explants showed minimal expression in the outer nuclear layer, high expression in the inner nuclear layer and very low expression within the ganglion cell layer, again similar to both $R L B P$ and PRKCA. CDK18, also known as Pctaire3, that is another poorly characterised member of the CDK5 family. It is expressed in neuronal tissue and there is evidence that it plays a role in the progression of Alzheimer's disease, since it is found in high concentrations in pathological tissue, and is proposed to modulate Tau phosphorylation (Herskovits and Davies 2006). More recently, it has been shown to prevent accumulated DNA damage and genome instability in response to replication stress (Barone et al. 2016).

In terms of distribution in the retina, all of the CDKs investigated were expressed in the INL, which could indicate expression in Müller cells. It is possible that CDK inhibition could affect these cells and since they are the major glial cells of the retina, providing essential support for the retinal neurons, therefore it was interesting to determine whether the pan-CDK inhibitor AG-012986 influences Müller cell survival. Exposure of MIO-M1 cells to AG-012986 for $24 \mathrm{~h}$ revealed a clear dose-dependent increase in cytotoxicity. Müller cells are therefore sensitive to inhibition of CDKs by AG-012986, with an increase in cell death, and not merely a decrease in proliferation. To clarify if the CDKs of interest were expressed in MIO-M1 cells, CDK5, 11, 16, 17 and 18 expression was measured. CDK11 expression was high in MIO-M1 cells. CDKs 5, 16 and 17 were also expressed, but expression of CDK18 was extremely low. Interestingly, the binding affinity data of the two neurotoxic CDK inhibitors both had a higher affinity for CDK11 than the non-toxic inhibitors. CDK11 might therefore be an interesting candidate for further investigation in relation to $\mathrm{CDK}$ inhibitor-induced death of Müller cells in relation to retinal toxicity.

\section{Conclusion}

This study aimed to investigate CDK expression in the human retina. Whole explant analysis and mRNA profiling from planar sectioned macula and paramacula explants revealed CDKs to be differentially expressed. CDK11 was predominantly expressed in the photoreceptor layer and the two neurotoxic CDK inhibitors tested were more potent at CDK11 than the non-neurotoxic CDK inhibitors. This research cannot confirm if CDK inhibitor-mediated photoreceptor toxicity may or may not occur in humans as has been shown in mice, however, CDK11 was found to be highly expressed in the human Müller cell line and exposure to the CDK inhibitor, AG-012986 caused cytotoxicity. It would be of interest to investigate the potential toxicity of AG-012986 in the ex vivo human retina, as this may clarify any potential clinically significant toxicity that the CDK inhibitor may possess. Correlation with the expression profiles determined here could give a better understanding of the mechanism of CDK-mediated retinotoxicity.

Acknowledgements The authors would like to express their gratitude to the staff of the East Anglian Eye Bank, especially Mary Tottman, and also Dr Matthew Peters at AstraZeneca. They also gratefully acknowledge funding from AstraZeneca and The Humane Research Trust.

Open Access This article is distributed under the terms of the Creative Commons Attribution 4.0 International License (http://creativeco mmons.org/licenses/by/4.0/), which permits unrestricted use, distribution, and reproduction in any medium, provided you give appropriate credit to the original author(s) and the source, provide a link to the Creative Commons license, and indicate if changes were made.

\section{References}

Bajic VP, Su B, Lee HG et al (2011) Mislocalization of CDK11/PITSLRE, a regulator of the G2/M phase of the cell cycle, in Alzheimer disease. Cell Mol Biol Lett 16(3):359-372. https://doi. org/10.2478/s11658-011-0011-2

Barone G, Staples CJ, Ganesh A et al (2016) Human CDK18 promotes replication stress signaling and genome stability. Nucleic Acids Res 44(18):8772-8785. https://doi.org/10.1093/nar/gkw615

Barsanti PA (2011) Pyridine and pyrzaine derivatives as protein kinase modulators. International Patent no. PCT/JP2008/073864 (WO/2011/012661)

Besset V, Rhee K, Wolgemuth DJ (1999) The cellular distribution and kinase activity of the Cdk family member Pctaire1 in the adult mouse brain and testis suggest functions in differentiation. Cell Growth Differ 10(3):173-181

Bregman DB, Pestell RG, Kidd VJ (2000) Cell cycle regulation and RNA polymerase II. Front Biosci 5:D244-D257 
Bringmann A, Pannicke T, Grosche J et al (2006) Muller cells in the healthy and diseased retina. Prog Retin Eye Res 25(4):397-424. https://doi.org/10.1016/j.preteyeres.2006.05.003

Chen J, Miao Y, Wang XH, Wang Z (2011) Elevation of p-NR2A(S1232) by Cdk5/p35 contributes to retinal ganglion cell apoptosis in a rat experimental glaucoma model. Neurobiol Dis 43(2):455-464. https://doi.org/10.1016/j.nbd.2011.04.019

Cheung ZH, Gong K, Ip NY (2008) Cyclin-dependent kinase 5 supports neuronal survival through phosphorylation of Bcl-2. J Neurosci 28(19):4872-4877. https://doi.org/10.1523/JNEUR OSCI.0689-08.2008

Di Giovanni C, Novellino E, Chilin A, Lavecchia A, Marzaro G (2016) Investigational drugs targeting cyclin-dependent kinases for the treatment of cancer: an update on recent findings (2013-2016). Expert Opin Investig Drugs 25(10):1215-1230. https://doi. org/10.1080/13543784.2016.1234603

Euler T, Haverkamp S, Schubert T, Baden T (2014) Retinal bipolar cells: elementary building blocks of vision. Nat Rev Neurosci 15(8):507-519

Fabian MA, Biggs WH, Treiber DK, Atteridge CE, Azimioara MD, Benedetti MG, Carter TA, Ciceri P, Edeen PT, Floyd M, Ford JM, Galvin M, Gerlach JL, Grotzfeld RM, Herrgard S, Insko DE, Insko MA, Lai AG, Lelias JM, Mehta SA, Milanov ZV, Velasco AM, Wodicka LM, Patel HK, Zarrinkar PP, Lockhart DJ (2015) A small molecule-kinase interaction map for clinical kinase inhibitors. Nat Biotechnol 23:329-336

Forrester JV (2007) The eye: basic sciences in practice, 3rd edition. Saunders, Philadelphia

Franco LC, Morales F, Boffo S, Giordano A (2018) CDK9: a key player in cancer and other diseases. J Cell Biochem 119(2):1273-1284. https://doi.org/10.1002/jcb.26293

Hayashi F, Matsuura I, Kachi S et al (2000) Phosphorylation by cyclindependent protein kinase 5 of the regulatory subunit of retinal cGMP phosphodiesterase. II. Its role in the turnoff of phosphodiesterase in vivo. J Biol Chem 275(42):32958-32965. https://doi. org/10.1074/jbc.M000703200

Herskovits AZ, Davies P (2006) The regulation of tau phosphorylation by PCTAIRE 3: implications for the pathogenesis of Alzheimer's disease. Neurobiol Dis 23(2):398-408. https://doi.org/10.1016/j. nbd.2006.04.004

Hirooka K, Tomizawa K, Matsui H et al (1996) Developmental alteration of the expression and kinase activity of cyclin-dependent kinase $5(\mathrm{Cdk} 5) / \mathrm{p} 35 \mathrm{nck} 5 \mathrm{a}$ in the rat retina. J Neurochem 67(6):2478-2483

Hirose T, Tamaru T, Okumura N, Nagai K, Okada M (1997) PCTAIRE 2, a Cdc2-related serine/threonine kinase, is predominantly expressed in terminally differentiated neurons. Eur J Biochem FEBS 249(2):481-488

Hu D, Mayeda A, Trembley JH, Lahti JM, Kidd VJ (2003) CDK11 complexes promote pre-mRNA splicing. J Biol Chem 278(10):8623-8629. https://doi.org/10.1074/jbc.M210057200

Huang CH, Lujambio A, Zuber J et al (2014) CDK9-mediated transcription elongation is required for MYC addiction in hepatocellular carcinoma. Genes Dev 28(16):1800-1814. https://doi. org/10.1101/gad.244368.114

Hydbring P, Malumbres M, Sicinski P (2016) Non-canonical functions of cell cycle cyclins and cyclin-dependent kinases. Nat Rev Mol Cell Biol 17(5):280-292. https://doi.org/10.1038/nrm.2016.27

Illanes O, Anderson S, Niesman M, Zwick L, Jessen BA (2006) Retinal and peripheral nerve toxicity induced by the administration of a pan-cyclin dependent kinase (cdk) inhibitor in mice. Toxicol Pathol 34(3):243-248. https://doi.org/10.1080/019262306007131 86

Jiang Z, Shen W (2010) Role of neurotransmitter receptors in mediating light-evoked responses in retinal interplexiform cells. J Neurophysiol 103(2):924-933. https://doi.org/10.1152/jn.00876.2009
Jones JA, Rupert AS, Poi M et al (2014) Flavopiridol can be safely administered using a pharmacologically derived schedule and demonstrates activity in relapsed and refractory non-Hodgkin's lymphoma. Am J Hematol 89(1):19-24. https://doi.org/10.1002/ ajh. 23568

Lameirao SV, Hamassaki DE, Rodrigues AR, SM DEL, Finlay BL, Silveira LC (2009) Rod bipolar cells in the retina of the capuchin monkey (Cebus apella): characterization and distribution. Visual Neurosci 26(4):389-396. https://doi.org/10.1017/S095252380 9990186

Lee DU, Jessen B (2012) Off-target immune cell toxicity caused by AG-012986, a pan-CDK inhibitor, is associated with inhibition of p38 MAPK phosphorylation. J Biochem Mol Toxicol 26(3):101108. https://doi.org/10.1002/jbt.20415

Li T, Weng T, Zuo M, Wei Z, Chen M, Li Z (2016) Recent progress of cyclin-dependent kinase inhibitors as potential anticancer agents. Future Med Chem. https://doi.org/10.4155/fmc-2016-0129

Lim S, Kaldis P (2013) Cdks, cyclins and CKIs: roles beyond cell cycle regulation. Development 140(15):3079-3093. https://doi. org/10.1242/dev.091744

Limb GA, Salt TE, Munro PM, Moss SE, Khaw PT (2002) In vitro characterization of a spontaneously immortalized human Muller cell line (MIO-M1). Investig Ophthalmol Vis Sci 43(3):864-869

Liu Y, Cheng K, Gong K, Fu AK, Ip NY (2006) Pctaire1 phosphorylates $N$-ethylmaleimide-sensitive fusion protein: implications in the regulation of its hexamerization and exocytosis. J Biol Chem 281(15):9852-9858. https://doi.org/10.1074/jbc.M513496200

Liu X, Cheng C, Shao B et al (2013) LPS-stimulating astrocyteconditioned medium causes neuronal apoptosis via increasing CDK11(p58) expression in PC12 cells through downregulating AKT pathway. Cell Mol Neurobiol 33(6):779-787. https://doi. org/10.1007/s10571-013-9945-4

Lu H, Xue Y, Yu GK et al (2015) Compensatory induction of MYC expression by sustained CDK9 inhibition via a BRD4-dependent mechanism. Elife 4:e06535. https://doi.org/10.7554/eLife.06535

Luke JJ, D'Adamo DR, Dickson MA et al (2012) The cyclin-dependent kinase inhibitor flavopiridol potentiates doxorubicin efficacy in advanced sarcomas: preclinical investigations and results of a phase I dose-escalation clinical trial. Clin Cancer Res 18(9):26382647. https://doi.org/10.1158/1078-0432.CCR-11-3203

Malumbres M (2014) Cyclin-dependent kinases. Genome Biol 15(6): 122

Malumbres M, Barbacid M (2005) Mammalian cyclin-dependent kinases. Trends Biochem Sci 30(11):630-641. https://doi. org/10.1016/j.tibs.2005.09.005

Matsuura I, Bondarenko VA, Maeda T et al (2000) Phosphorylation by cyclin-dependent protein kinase 5 of the regulatory subunit of retinal cGMP phosphodiesterase. I. Identification of the kinase and its role in the turnoff of phosphodiesterase in vitro. J Biol Chem 275(42):32950-32957. https://doi.org/10.1074/jbc.M000702200

Mita MM, Joy AA, Mita A et al (2014) Randomized phase II trial of the cyclin-dependent kinase inhibitor dinaciclib (MK-7965) versus capecitabine in patients with advanced breast cancer. Clin Breast Cancer 14(3):169-176. https://doi.org/10.1016/j.clbc.2013.10.016

Nakayama T, Goshima Y, Misu Y, Kato T (1999) Role of cdk5 and tau phosphorylation in heterotrimeric $\mathrm{G}$ protein-mediated retinal growth cone collapse. J Neurobiol 41(3):326-339

Nemunaitis JJ, Small KA, Kirschmeier P et al (2013) A first-in-human, phase 1 , dose-escalation study of dinaciclib, a novel cyclindependent kinase inhibitor, administered weekly in subjects with advanced malignancies. J Transl Med 11:259. https://doi. org/10.1186/1479-5876-11-259

Niyadurupola N, Sidaway P, Osborne A, Broadway DC, Sanderson J (2011) The development of human organotypic retinal cultures (HORCs) to study retinal neurodegeneration. Br J Ophthalmol 95(5):720-726. https://doi.org/10.1136/bjo.2010.181404 
Niyadurupola N, Sidaway P, Ma N, Rhodes JD, Broadway DC, Sanderson J (2013) P2X7 receptor activation mediates retinal ganglion cell death in a human retina model of ischemic neurodegeneration. Invest Ophthalmol Vis Sci 54:2163-2170

Okuda H, Takahashi S, Takaori-Kondo A, Yokoyama A (2016) TBP loading by AF4 through SL1 is the major rate-limiting step in MLL fusion-dependent transcription. Cell Cycle 15(20):27122722. https://doi.org/10.1080/15384101.2016.1222337

Osborne A, Hopes M, Wright P, Broadway DC, Sanderson J (2016) Human organotypic retinal cultures (HORCs) as a chronic experimental model for investigation of retinal ganglion cell degeneration. Exp Eye Res 143:28-38. https://doi.org/10.1016/j. exer.2015.09.012

Parry D, Guzi T, Shanahan F et al (2010) Dinaciclib (SCH 727965), a novel and potent cyclin-dependent kinase inhibitor. Mol Cancer Ther 9(8):2344-2353. https://doi.org/10.1158/1535-7163. MCT-10-0324

Petretti C, Savoian M, Montembault E, Glover DM, Prigent C, Giet R (2006) The PITSLRE/CDK11p58 protein kinase promotes centrosome maturation and bipolar spindle formation. EMBO Rep 7(4):418-424. https://doi.org/10.1038/sj.embor.7400639

Poche RA, Reese BE (2009) Retinal horizontal cells: challenging paradigms of neural development and cancer biology. Development 136(13):2141-2151. https://doi.org/10.1242/dev.033175

Rahl PB, Lin CY, Seila AC et al (2010) c-Myc regulates transcriptional pause release. Cell 141(3):432-445. https://doi.org/10.1016/j. cell.2010.03.030

Rakkaa T, Escude C, Giet R, Magnaghi-Jaulin L, Jaulin C (2014) CDK11(p58) kinase activity is required to protect sister chromatid cohesion at centromeres in mitosis. Chromosome Res Int J Mol Supramol Evol Asp Chromosome Biol 22(3):267-276. https://doi. org/10.1007/s10577-013-9400-x

Reichenbach A, Bringmann A (2013) New functions of Muller cells. Glia 61(5):651-678. https://doi.org/10.1002/glia.22477

Samarakkody A, Abbas A, Scheidegger A et al (2015) RNA polymerase II pausing can be retained or acquired during activation of genes involved in the epithelial to mesenchymal transition. Nucleic Acids Res 43(8):3938-3949. https://doi.org/10.1093/ nar/gkv263

Sanes JR, Masland RH (2015) The types of retinal ganglion cells: current status and implications for neuronal classification. Annu Rev Neurosci 38:221-246. https://doi.org/10.1146/annurev-neuro $-071714-034120$

Shimizu K, Uematsu A, Imai Y, Sawasaki T (2014) Pctaire1/Cdk16 promotes skeletal myogenesis by inducing myoblast migration and fusion. FEBS Lett 588(17):3030-3037. https://doi.org/10.1016/j. febslet.2014.05.060

Stephenson JJ, Nemunaitis J, Joy AA et al (2014) Randomized phase 2 study of the cyclin-dependent kinase inhibitor dinaciclib (MK7965) versus erlotinib in patients with non-small cell lung cancer. Lung Cancer 83(2):219-223. https://doi.org/10.1016/j.lungc an.2013.11.020

Su SC, Tsai LH (2011) Cyclin-dependent kinases in brain development and disease. Annu Rev Cell Dev Biol 27:465-491. https://doi. org/10.1146/annurev-cellbio-092910-154023

Sutton J (2014) Selective CDK9 inhibitors: stories in lead optimization and toxicology. In: American Association for Cancer Research meeting webcast. http://webcast.aacr.org/console/player/22617 ?mediaType $=$ slideVideo $\&$. Accessed 03 Oct 2018

Wilkinson S, Croft DR, O'Prey J et al (2011) The cyclin-dependent kinase PITSLRE/CDK11 is required for successful autophagy. Autophagy 7(11):1295-1301. https://doi.org/10.4161/ auto.7.11.16646

Yanagi T, Reed JC, Matsuzawa S (2014) PCTAIRE1 regulates p27 stability, apoptosis and tumor growth in malignant melanoma. Oncoscience 1(10):624-633. https://doi.org/10.18632/oncoscience.86

Zhang J, Zhou L, Zhao S, Dicker DT, El-Deiry WS (2017) The CDK4/6 inhibitor palbociclib synergizes with irinotecan to promote colorectal cancer cell death under hypoxia. Cell Cycle 16(12):11931200. https://doi.org/10.1080/15384101.2017.1320005 